\title{
Development of a DMD-based fluorescence microscope
}

\author{
Nadya Chakrova, Bernd Rieger, Sjoerd Stallinga \\ Delft University of Technology, Lorentzweg 1, 2628CJ, Delft, The Netherlands
}

\begin{abstract}
We present a versatile fluorescence microscope, built by complementing a conventional fluorescence microscope with a digital micro-mirror device (DMD) in the illumination path. Arbitrary patterns can be created on the DMD and projected onto the sample. This patterned illumination can be used to improve lateral and axial resolution over the resolution of a wide-field microscope, as well as to reduce the illumination dose. Different illumination patterns require different reconstruction strategies and result in an image quality similar to confocal or structured illumination microscopy. We focus on the optical design and characterization of a DMD-based microscope. Estimation of the optical quality of the microscope has been carried out by measuring the modulation transfer function from edge profiles. We have obtained optically sectioned images by applying multi-spot illumination patterns followed by digital pinholing. The sectioning capabilities of our DMD-based microscope were estimated from the dependence of the signal-to-background and signalto-noise ratios on the pitch of the projected multi-spot patterns and the size of the digital pinhole. In addition, we provide an outlook on the use of pseudo-random illumination patterns for achieving both sectioning and resolution enhancement.
\end{abstract}

Keywords: fluorescence microscopy, digital micro-mirror device, sectioning, digital pinholing

\section{INTRODUCTION}

Fluorescence microscopy serves biologists as an imaging technique for studying intracellular processes and structures. However, it is well known, that the resolution of a standard wide-field microscope is limited by diffraction and the smallest resolved periodical structure is defined by Abbe's formula as $\lambda / 2 \mathrm{NA}$, where $\lambda$ is the wavelength and NA is the numerical aperture of the objective lens. In the last two decades, attempts to overcome this resolution limit led to various fluorescence microscopy techniques, providing lateral resolutions in the range of $\sim 20-150 \mathrm{~nm}$ and axial resolutions in the range of $\sim 100-700 \mathrm{~nm}$ (see Ref. 1 for an overview). Unfortunately, none of these methods is universal and an appropriate technique has to be chosen for each particular application to achieve the desired trade-off between the resolution improvement, signal-to-noise ratio, imaging time and photo-bleaching.

In fluorescence microscopy, non-uniform illumination can be used to improve lateral resolution, provide sectioning and reduce the illumination dose. Most prominent examples of the microscopy techniques that utilize nonuniform illumination are confocal microscopy ${ }^{2}$ and structured-illumination microscopy (SIM) ${ }^{3-7}$. In confocal microscopy, sectioning is achieved by using point illumination and detection. The speed of image acquisition can be increased by using parallel scanning techniques, such as spinning disk microscopy. In SIM periodic line patterns, which are created by the interference of two or three laser beams in the sample plane, are used to double the resolution. The final image has to be reconstructed from several images acquired at different positions and orientations of the line pattern. Apart from point illumination and periodic line pattern illumination, sequences of pseudo-random patterns have been studied in spinning disk systems to address the problem of inefficient use of the available illumination light ${ }^{8}$.

The use of spatial light modulators, such as liquid crystal-on-silicon (LCOS) micro-displays or digital micromirror devices (DMD) provides spatial and temporal control over the illumination patterns and allows to combine all the mentioned patterned illumination techniques in one setup. Such a microscope is expected to produce images with a quality similar to confocal or SIM microscopy, depending on the type of projected illumination pattern. Moreover, spatial light modulators can be used to reduce the illumination dose by controlling the illumination pattern pixel by pixel $^{9}$. Together with improvements in LCOS and DMD technologies, a number of DMD-based and LCOS-based microscopes were developed ${ }^{10-16}$. Although the performance of the DMD-based and LCOS-based microscopes is very similar, we consider the use of the DMD advantageous, due to its higher switching speed and higher contrast over a larger spectral range.

In the following sections we focus on the optical design and characterization of DMD-based microscopes. We first study the DMD operation and estimate the optical quality of the microscope by measuring its modulation transfer

Three-Dimensional and Multidimensional Microscopy: Image Acquisition and Processing XXII, edited by

Thomas G. Brown, Carol J. Cogswell, Tony Wilson, Proc. of SPIE Vol. 9330, 933008 - (c) 2015 SPIE .

CCC code: $1605-7422 / 15 / \$ 18 \cdot$ doi: $10.1117 / 12.2077677$ 
function (MTF). Next, we present the examples of sectioned images, produced by projecting the multi-spot patterns onto the sample, and estimate the sectioning by measuring signal-to-background and signal-to-noise ratios. In conclusion, we give an outlook on the use of pseudo-random illumination patterns for achieving both sectioning and resolution enhancement.

\section{EXPERIMENTAL METHODS}

\subsection{Digital micro-mirror device operation}

A digital micro-mirror device (DLP discovery 4100 chip set, Texas Instruments, TX, USA) consists of an array of $1024 \times 768$ square micro-mirrors with a pitch of $13.68 \mu \mathrm{m}$ and the physical size of the mirror somewhat less due to the fill factor of $91 \%$. Each micro-mirror can be tilted along its diagonal to occupy one of two positions: $+12^{\circ}$ tilt to direct the incident beam into the optical path, $-12^{\circ}$ tilt to deflect the incident beam away from the optical path. Each pixel of the DMD is addressed separately, thus providing the possibility to create arbitrary illumination patterns. Since the mirrors of the DMD tilt along their diagonals, the DMD chip is rotated $45^{\circ}$ in order to keep both incident and reflected beams in one plane, parallel to the optical table. The DMD is controlled by the ALP-4.2 Controller Suite (Vialux, Chemnitz, Germany), which is a hardware, firmware and software package, that allows for a $22 \mathrm{kHz}$ switching rate of the binary patterns.

Particular diffraction characteristics of the DMD have to be understood prior to optimal use of this device in an imaging system. The pixelated structure of the DMD is essentially a two-dimensional blazed diffraction grating with pitch $d=d_{D M D} / \sqrt{ } 2$ and a facet tilt angle of $\theta=12^{\circ}$. In a blazed grating, intensity of the diffracted light for wavelength $\lambda$ in case of normal incidence is distributed among $m$ diffraction orders with angles $\beta_{m}=\arcsin (m \lambda / d)$, and the center of the intensity distribution is not coupled with the 0 -order ${ }^{17}$. When $\beta_{m}=2 \theta$, the blaze condition is fulfilled and the order $m$ receives most of the diffraction intensity. For given incidence angle and grating parameters each diffraction order can be at blaze condition for only one wavelength. Table 1 shows the blazed orders and the corresponding blazed wavelengths in case of the normal incidence for the DMD used in this work.

Table 1. Blazed orders and corresponding wavelengths in case of the normal incidence.

\begin{tabular}{|c|c|}
\hline Blazed order & Wavelength, $\mathrm{nm}$ \\
\hline 6 & 656 \\
\hline 7 & 562 \\
\hline 8 & 492 \\
\hline 9 & 437 \\
\hline 10 & 394 \\
\hline
\end{tabular}

In the general case, the illumination wavelength does not satisfy the blazed grating condition and diffracted light from the DMD is not parallel to the optical axis ${ }^{18}$. Fig.1 shows this effect for a few typical laser wavelengths. The top row presents simulated diffraction intensities at the conjugate pupil plane of the microscope. The simulation is based on the mathematical description of the diffraction amplitude at the DMD given in reference 19. The bottom row shows images of the DMD diffraction patterns, projected on a white screen, taken with a digital consumer camera. Small white circles in the top row and small black circles in the bottom row represent typical numerical aperture at the image plane of the microscope $\mathrm{NA}=0.012$. One can notice, that without taking additional measures, some wavelengths will not be transmitted through the light path of the microscope with such an NA. The angle of incidence of the illumination beam has to be tuned to match the blazed grating condition for a specific wavelength.

In case an additional, identical DMD is also used at the detection side, tuning of the incidence angle to achieve blazed condition is not possible, as excitation and emission wavelengths differ due to the Stokes shift. One of the solutions to this problem is to increase the numerical aperture of the relay optics between the DMD and camera in order to capture enough light even in the off-blaze condition ${ }^{18}$. To estimate the required NA, we refer again to the mathematical description of the diffraction amplitude at the DMD. In the off-blaze case, each of the four closest diffraction orders receives $16 / \pi^{4} \approx 16 \%$ of the diffracted light intensity. Ensuring that at least these closest diffraction orders in the off-blaze case are captured guarantees a total light efficiency of $64 \%$ or higher for any wavelength. In 
practice, that means increasing the NA $\sim 4$ times in comparison to the typical values of NA in the microscope's image plane. Large white circles in Fig.1 represent this increased numerical aperture NA $=0.05$.

In this work we have used a DMD only at the illumination side and tuned the incidence angle of the diode laser beam with $488 \mathrm{~nm}$ wavelength to meet the blazing condition. A pinhole placed in the Fourier plane of the DMD blocks all the diffraction orders except for the central one, in order to avoid diffraction induced distortions of the illumination pattern.

We have observed a mismatch in the intensities of the modelled and imaged diffraction patterns. Particularly, intensities of the diffraction spots in the imaged patterns exhibit top-bottom asymmetry, which is not predicted by the theory. We have not identified the reason for this asymmetry, however, we note that it was persistent with changes in polarization and alignment.
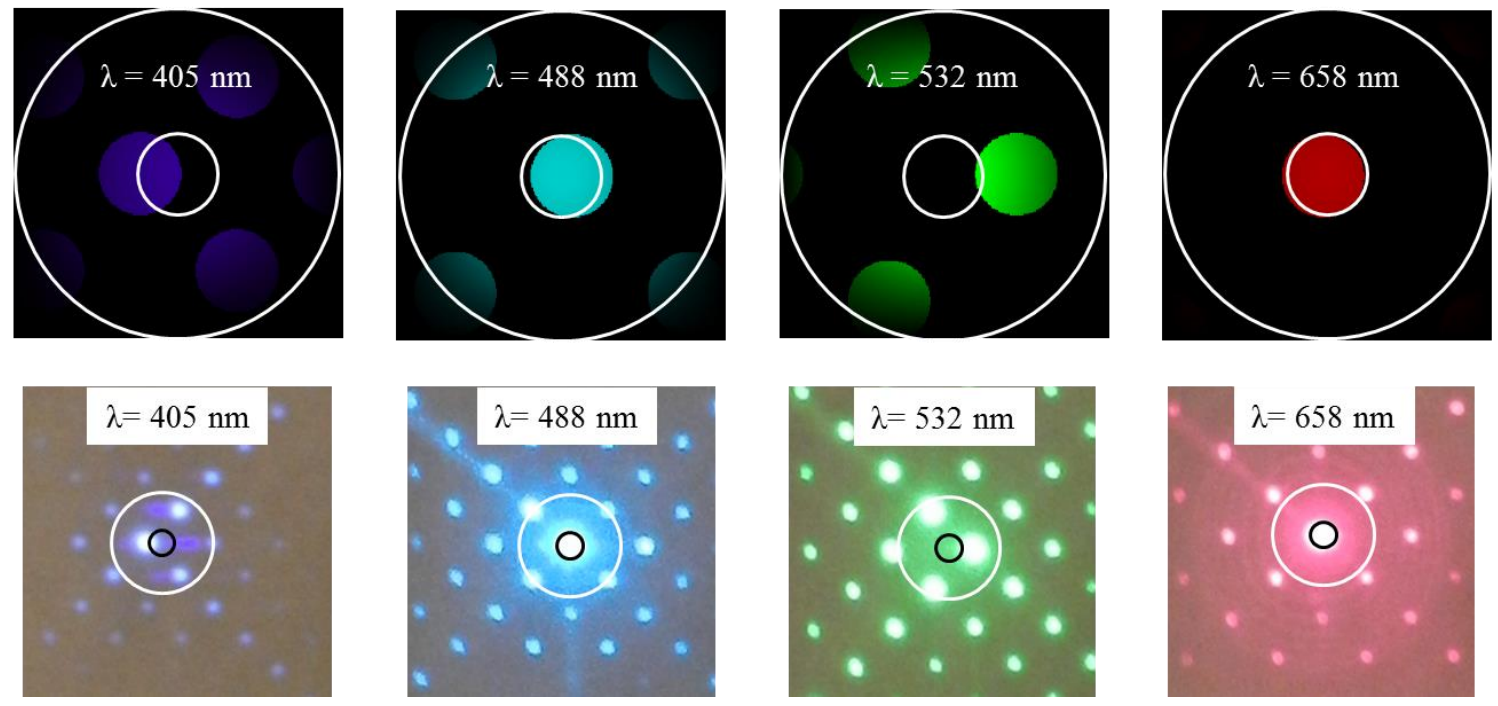

Figure 1. Simulated (top row) and imaged (bottom row) diffraction intensities at the conjugate pupil plane of the microscope for different wavelengths. Small white circles in the top row and small black circles in the bottom row represent typical numerical aperture at the image plane of the microscope $\mathrm{NA}=0.012$. Large white circles in the top and bottom rows represent increased numerical aperture NA $=0.05$.

\subsection{Setup layout}

A DMD-based fluorescence microscope is built on the basis of an inverted Olympus IX71 microscope. The DMD is placed in a plane conjugate to the image plane of the microscope. The lens relay from the DMD to the microscope image plane is made from two achromatic doublets and has a magnification of 1.66x. Using a 100x oil immersion objective each DMD pixel of $13.68 \times 13.68 \mu \mathrm{m}$ is demagnified $1.66 \times 100=166$ times down to $83 \times 83 \mathrm{~nm}$ in the sample plane. The DMD is illuminated by a $100 \mathrm{~mW}$ diode laser source with $488 \mathrm{~nm}$ wavelength (Coherent Inc., CA, USA). The laser beam is first despeckled by a rotating diffuser (SUSS MicroOptics, Switzerland) and expanded to the size that covers the full area of the DMD. The expanded and despeckled laser beam is then directed to the DMD at an angle that matches the blazed grating condition of the DMD for $488 \mathrm{~nm}$ wavelength. The U-MWIB2 Olympus filter cube with ET535/50M dichroic mirror, which is used to separate the excitation and emission bands, is placed between the tube lens and the image plane. Placing the filter cube outside of the parallel light path is generally avoided in microscopy, since it can introduce additional distortions to the optical system. In our case the filter cube is placed outside of the parallel path to enable the design where both excitation and emission beams pass through the side port of the microscope. Fluorescence from the sample is imaged by an sCMOS camera (Hamamatsu Photonics, Hamamatsu City, Japan) placed at the image plane of the microscope. The optical layout of the experimental setup is given in Fig.2. In order to provide proper sampling, two Nyquist criteria have to be satisfied - Nyquist sampling by the DMD is achieved if condition (1) is fulfilled, and Nyquist sampling by the camera is achieved if condition (2) is fulfilled.

$$
d_{D M D} \leq \lambda_{e x} / 4 N A
$$




$$
d_{\text {SCMOS }} \leq \lambda_{\text {em }} / 4 N A
$$

If a $100 \times$ objective with $\mathrm{NA}=1.3$ is used, the required DMD pixel size in the object plane equals $94 \mathrm{~nm}$ and the required sCMOS pixel size equals $100 \mathrm{~nm}$, assuming a Stokes shift to $518 \mathrm{~nm}$. Hence, the actual DMD pixel size of $83 \mathrm{~nm}$ and the actual sCMOS pixel size of $65 \mathrm{~nm}$ provide slight oversampling of the object plane.

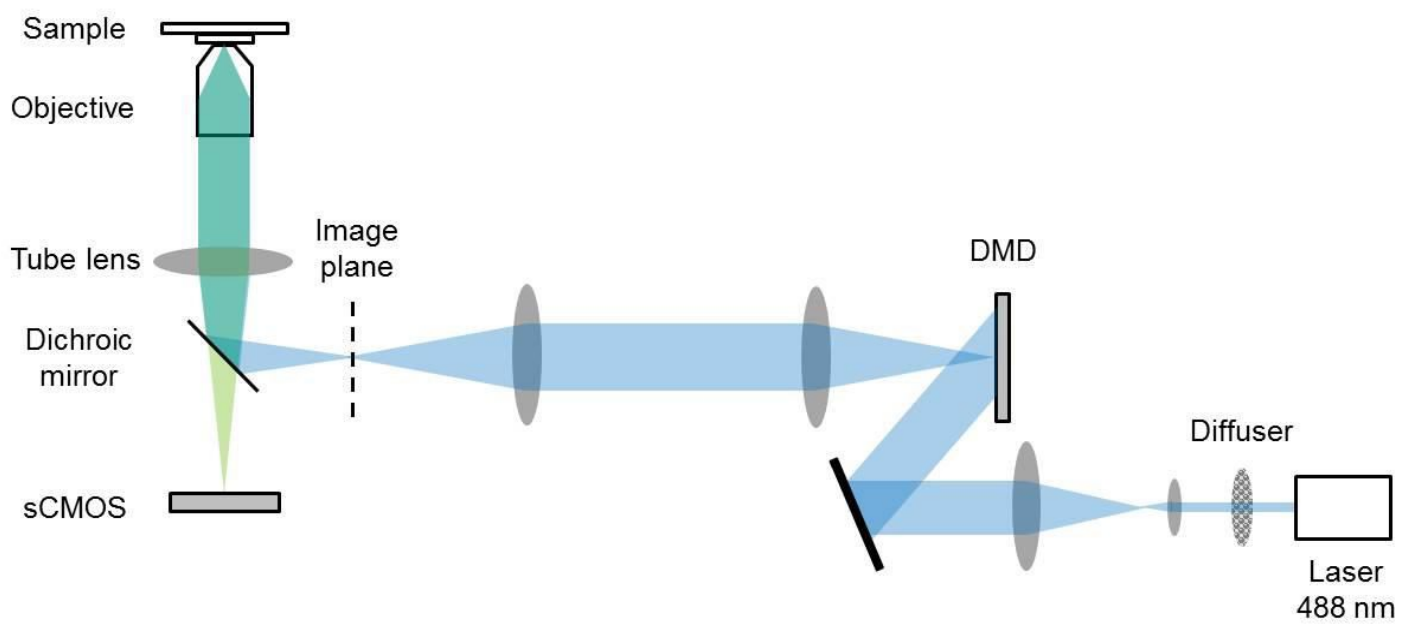

Figure 2. Schematic view of the setup. The DMD is illuminated by the expanded and despeckled $488 \mathrm{~nm}$ laser beam. The pattern of the DMD is projected into the image plane via a $1.66 \times$ lens relay and further into the sample plane via the tube lens and the objective of the microscope. Fluorescence of the sample passes through the dichroic mirror and is imaged by the sCMOS camera.

\subsection{Samples}

A thin fluorescent layer was used for the calibration of the system by edge profile and noise measurements and flat field images. The layer is $c a .110 \mathrm{~nm}$ thick and exhibits high spatial fluorescence homogeneity. The layer was originally developed to characterize the image quality in sectioning microscopy and is produced as described in Ref. 20.

Multi-spot scans were applied to the bovine pulmonary artery endothelial (BPAE) cells (Life Technologies, CA, USA), in which F-actin is stained with Alexa Fluor 488 phalloidin.

\section{OPTICAL QUALITY ASSESSMENT}

\subsection{Estimating the MTF by the edge-profile measurement method}

One of the ways to estimate the resolution of an optical system is to measure its Modulation Transfer Function (MTF). In order to estimate the MTF, we have performed edge-profile measurements (see Fig.3 and Ref. 21). First, a sharp edge pattern was created on the DMD by switching half of the pixels in the "on"-state and another half in the "off"-state. The edge patterns were projected into the thin, homogeneously fluorescent layer as described above, and the images were captured by the sCMOS camera. The image of the edge on the camera is the Edge Spread Function (ESF) - the twodimensional convolution of the edge-source object with the excitation and emission PSF of the system. The camera and the DMD are precisely aligned in such a way that the DMD edge is strictly vertical or strictly horizontal with respect to the camera chip. The edge profile was calculated from an average of 10 images. After dark-field and flat-field correction, we averaged the resulting image over several hundreds of camera rows to extract the vertical edge or over several hundreds of columns to extract the horizontal edge. The obtained edge profile is a one-dimensional projection of the ESF. Next, the ESF was differentiated in order to get the one-dimensional projection of the Line Spread Function (LSF). Finally, the MTF curve was found as the Fourier transform of the LSF. 

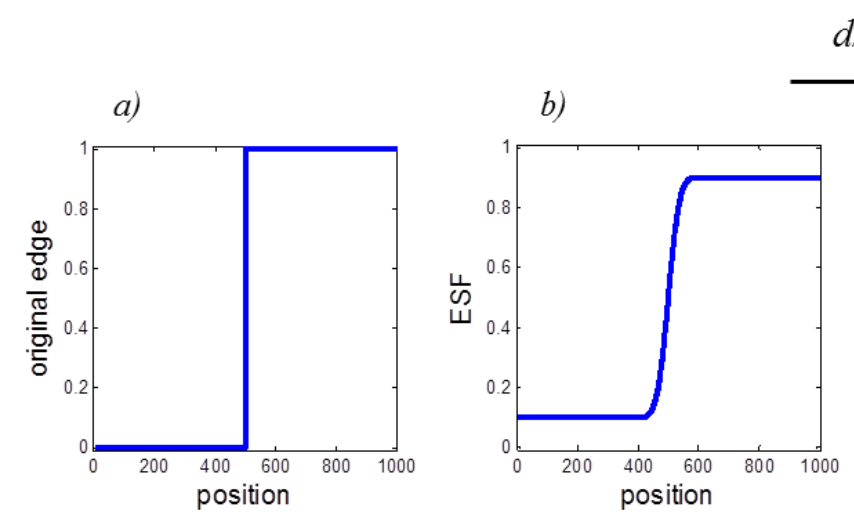

$d / d x$

FT
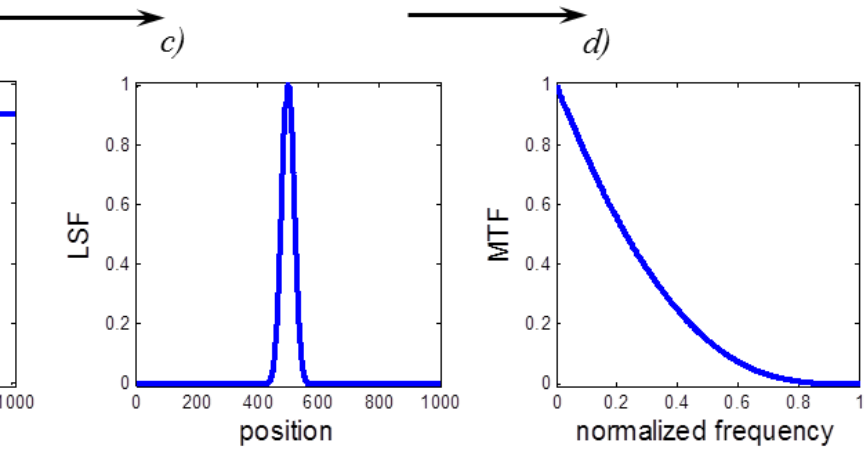

Figure 3. Estimation of the MTF from the edge-profile measurement. a) Original sharp edge b) Edge Spread Function c) Line-Spread Function d) Modulation Transfer Function.

In order to reduce the noise in the MTF curves, the following procedure was applied. The edge profile was upsampled by a factor of 8 using spline interpolation. Noise on the high and low plateaus of the edge profile was reduced by calculating a moving average over a varying window. After differentiation by finite differences, the LSF was downsampled back to its original rate and the zero level of the LSF was further smoothed down by applying the moving average over a varying window.

\subsection{Theoretical MTF curves}

The MTF of an incoherent optical system can be calculated as the normalized autocorrelation of the exit pupil of the system. In case of a circular aperture the MTF is of the form:

$$
\operatorname{MTF}\left(\frac{f}{f_{\text {cutoff }}}\right)=\frac{2}{\pi}\left(\cos ^{-1}\left(\frac{f}{f_{\text {cutoff }}}\right)-\left(\frac{f}{f_{\text {cutof } f}}\right) \sqrt{1-\left(\frac{f}{f_{\text {cutoff }}}\right)^{2}}\right),
$$

where the cutoff frequency $f_{\text {cutoff }}=2 N A / \lambda$, is the highest frequency transmitted by the diffraction-limited system with numerical aperture NA for a given wavelength $\lambda^{22}$.

In our experiment, a sharp edge is created as a pattern on the DMD and projected into the thin fluorescent layer by the microscope objective. The projected edge is then imaged on the sCMOS camera by the same microscope objective. Since we are imaging not the sharp edge directly, but the image of this edge, each point of the original edge is blurred twice by passing twice through the optical path. Hence, the MTF given by formula (3) has to be squared in order to correctly represent the expected optical MTF. The MTF of the camera is found as a Fourier transform of the camera pixel. The pixel shape is represented by the rectangle function and its Fourier transform - by the sinc function:

$$
M T F_{\text {camera }}=\operatorname{sinc}(\pi a f) \text {, }
$$

with $a$ the pixel size of the camera in the sample plane. The final theoretical MTF is calculated as a product of the optical MTF and camera MTF.

\subsection{Comparison of the experimental and theoretical MTF curves}

MTF curves were measured for two objectives: $10 \times$ air objective with NA $=0.25$ and $100 \times$ oil immersion objective with $\mathrm{NA}=1.3$. We have imaged vertical and horizontal edges of two types. Owing to the structure of the DMD, edges of type 1 are formed by neighboring pixels at an angle of $156^{\circ}$ and edges of type 2 are formed by neighboring pixels at an angle of $204^{\circ}$ (Fig.4). This difference in the geometrical structure of the edges influences the edge profiles of the system and results in different MTF curves for different edge types.

Measured MTF curves for the $10 \times$ objective correspond well to the theoretical MTFs, which indicates that the microscope performance is close to diffraction-limited in this case (Fig.5). High NA objectives are more complex and are more sensitive to misalignments in the system. The measured MTF curves for the $100 \times$ oil immersion objective are 
also sensitive to the edge type, as can be seen from Fig.6. Edge type 1 results in higher values for the MTF than edge type 2. The difference in the MTF curves is the result of the difference in the geometrical structure of the edges at the DMD per se. Noteworthy, this difference is not prominent in the MTF curves for the low NA objective.

We have measured the MTF with an alternative method in order to avoid this edge-type sensitivity in the MTF curves. Horizontal and vertical lines with a thickness of a single DMD pixel where projected into the sample. Images of these projections correspond to the LSF profiles. Taking the Fourier transform of the LSF results in an edge-type independent MTF measurement, as shown in Fig.6.

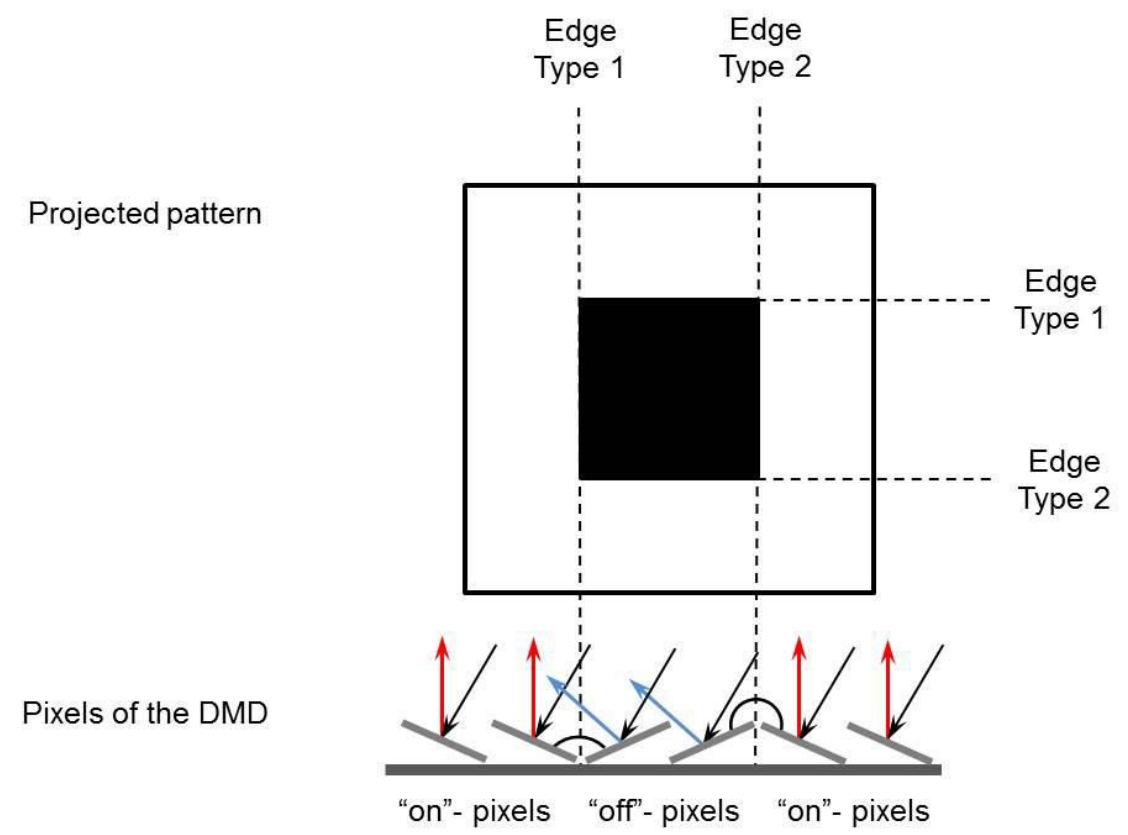

Figure 4. The edges formed by the DMD. Black arrows indicate the incident light, red arrows - light reflected by the pixels in the "on" state, blue arrows - light, reflected by the pixels in the "off" state.
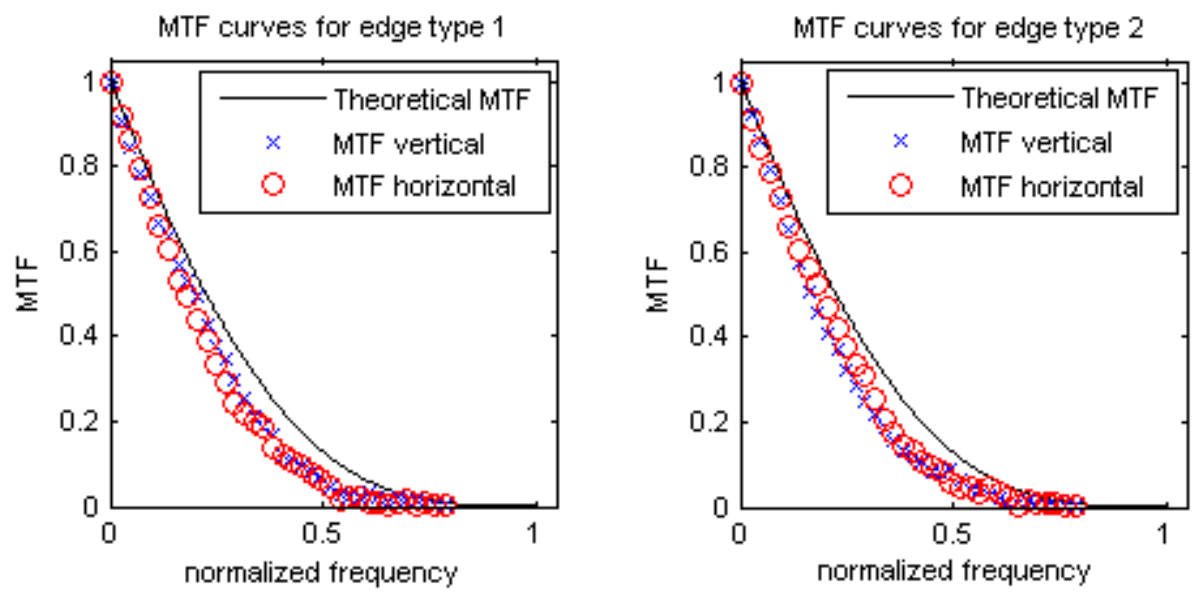

Figure 5. The MTF curves for $10 \times$ objective with $\mathrm{NA}=0.25$ indicate than the microscope performance is close to diffraction-limited. The spatial frequency is normalized to the cutoff spatial frequency $f_{\text {CUTOFF }}=2 N A / \lambda$. 

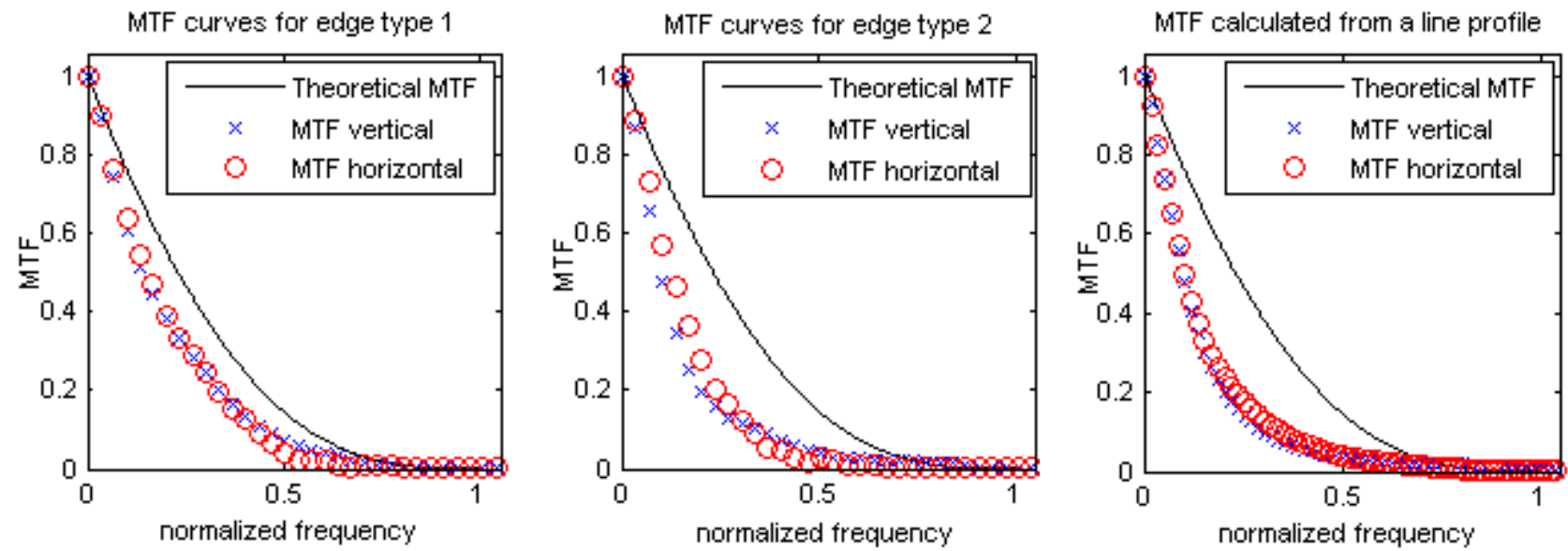

Figure 6. The MTF curves for $100 \times$ objective with NA $=1.3$. Edge type 1 results in higher MTF values than edge type 2. The MTF profiles differ due to the difference in the edges of the DMD per se. MTF curve calculated from a line profile shows an edge-independent MTF measurement.

\section{MAPPING THE DMD TO THE CAMERA}

Each pixel of the DMD that is projected into the sample, appears as a blurred spot in the raw camera image. In order to apply reconstruction algorithms, we have to establish the correspondence between each DMD-pixel and its image on the camera. To create a precise mapping of the DMD pixels onto the camera grid, we apply the following procedure. A calibration image is taken by projecting a multi-spot DMD pattern into a thin, homogeneously fluorescent layer. The multi-spot pattern is created as an array of switched "on" DMD pixels, arranged in a square grid with pitch $p$. The square with the side that equals the pitch size of the projected grid is called the unit cell. Assuming that the unit cells which are closest to the optical axis are not distorted in the camera image, we find the unit cell vector $c$ as best fitting vector (in the least squares sense) to the $6 \times 6$ central unit cells. The square grid with the pitch size of the unit cell vector is the grid of expected spot positions of the DMD-pixels in the camera $\left(x_{c}, y_{c}\right)$. Actual spot positions deviate from this grid of expected positions due to distortions in the system. This deviation increases with increasing distance from the optical axis. The actual spot positions $\left(x_{c}, y_{c}{ }^{\prime}\right)$ are found with subpixel precision as local maxima in the neighbourhoods of the expected spots positions $\left(x_{c}, y_{c}\right)$. In order to find the coordinates of all the remaining pixels of the DMD in the camera plane, the found positions of the actual spots of the square grid have to be translated $p^{2}-1$ times with the step that equals $c / p$. Thus, each DMD pixel $\left(x_{d m d}, y_{d m d}\right)$ is mapped on the camera grid with subpixel precision as $\left(x_{c}, y_{c}{ }^{\prime}\right)$. An example of the spot detection in a calibration image is given in Fig. 7.

The deviation of the actual spots from the expected spots provides valuable information about distortions that are present in the system. For our reconstruction procedure we have used the part of the image field of view (FOV) where the actual spot positions deviated from the expected spot positions by less than 1.5 camera pixels. Such a FOV typically amounts to approximately $800 \times 800$ camera pixels, which corresponds to an area of $52 \mu \mathrm{m} \times 52 \mu \mathrm{m}$ in the sample plane if a $100 \times$ oil immersion objective is used. 


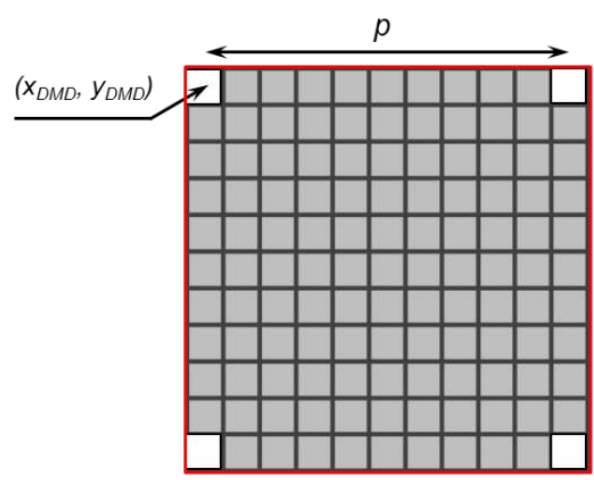

unit cell $p \times p$ at the DMD

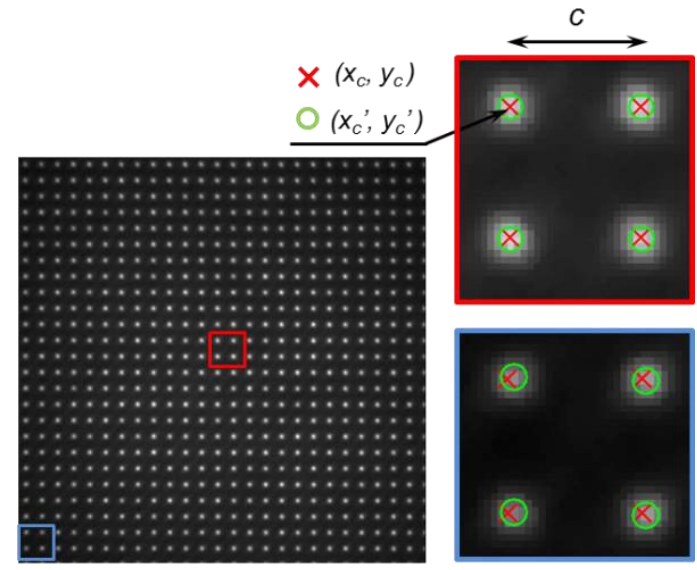

calibration image

Figure 7. Mapping of the DMD to the camera is performed by detecting the illumination spots in the calibration image. Each DMD pixel $\left(x_{D M D}, y_{D M D}\right)$ is mapped onto the camera grid with subpixel precision as $\left(x_{c}{ }^{\prime}, y_{c}{ }^{\prime}\right)$.

\section{MULTI-SPOT ILLUMINATION RESULTS}

We obtained optically sectioned images by projecting multi-spot patterns onto the sample and applying digital pinholing to the raw images. First, a square grid pattern with pitch $p$ is projected onto the sample. To illuminate the sample homogeneously, the pattern is translated $p^{2}-1$ times with a translation step of one DMD pixel. A camera image is taken for each pattern translation, so that in total $p^{2}$ camera frames are collected. After dark field and flat field correction, we apply digital pinholing for each raw camera frame to suppress the out-of-focus light. Digital pinholing is performed by detecting the spots in each camera frame and multiplying each of the spots with a 2D Gaussian mask with standard deviation $\sigma$. The value of $\sigma$ controls the strength of the sectioning effect as will be shown later in this section. For each Gaussian spot, the intensities of all the pixels falling within a spot are summed up, and the resulting intensity value is assigned to the corresponding DMD pixel. In this way, each raw camera image is converted into an image that has the number of pixels of the DMD chip. Accurate mapping of the DMD to the camera is essential to perform this step. After the pinholing, $p^{2}$ converted images are summed up to build an optically sectioned image. A comparison of such an image with a standard wide-field image is given in Fig. 8.

Two main parameters influencing the sectioning capabilities of a DMD-based microscope for a given objective are the pitch $p$ of the projected pattern and the pinholing Gaussian mask width $\sigma$. By changing the pitch we are changing the trade-off between the speed of image acquisition, which is given by the number of needed raw frames, and the sectioning strength (Fig. 9a, 10). The optimal pitch should provide the smallest number of raw images without compromising the sectioning quality. For the parameters of our setup, $p=12-16$ DMD pixels proved to be sufficient to this end. Smaller pinhole separations result in a significant crosstalk between the pinholes and hamper the detection of the individual illumination spots, which results in artefacts in the reconstructed image. Larger pinhole separations increase the imaging time without providing substantially better sectioning. The sectioning effect can be assessed by the background level in the reconstructed images, since a better sectioning results in a higher signal-to-background ratio (SBR). The SBR was estimated by analysing the images of the BPAE cells, reconstructed after the multi-spot illumination. We have estimated the signal as the mean of the $1 \%$ highest intensity pixels and the background as the mean of the background area. The resulting SBR in the reconstructed image with $p=16$ DMD pixels is approximately 6 times higher than the SBR in the widefield image. By squeezing the digital pinhole parameter $\sigma$ we enhance the sectioning, but at the same time reduce the signal-to-noise ratio (SNR) (Fig. 9b, 10). The SBR was estimated in the same manner as previously. The SNR was measured by analysing images of the thin homogeneously fluorescent layer. After dark field and flat field correction, the signal was measured as the mean intensity of the image, and the noise as the standard deviation of the intensity in the image. In confocal microscopy the size of the digital pinhole is usually set as the diameter of the first dark ring of the Airy pattern $d_{\text {Airy }}$. A Gaussian profile corresponding to the Airy pattern with $d_{\text {Airy }}$ $=1.22 \lambda / \mathrm{NA}$ has a standard deviation $\sigma \approx 0.45 \lambda / 2 \mathrm{NA} \approx 85 \mathrm{~nm}$ for the given objective. Horizontal lines in Fig. 9 
correspond to the SBR and SNR in a widefield image. The illumination dose used for the widefield image was approximately 10 times lower than the total illumination dose that was used for the multi-spot scan. Therefore, the SNR of the widefield image was scaled by the factor of $\sqrt{10}$ in order to compare the widefield and the reconstructed images at equal illumination doses in Fig. $9 \mathrm{~b}$.
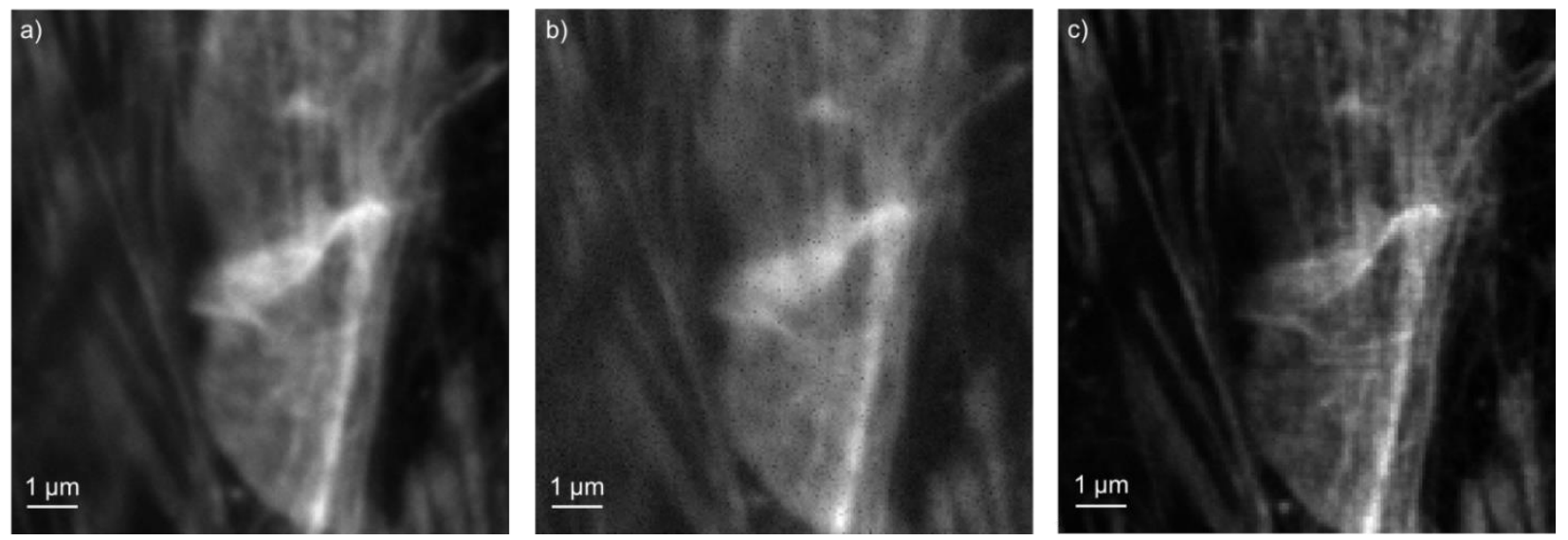

Figure 8. Images of BPAE cells stained with phalloidin conjugated to Alexa Fluor 488, acquired with a $100 \times / 1.3$ objective. a) Widefield image b) The sum of $p^{2}$ frames acquired at all scanning positions of the multi-spot illumination. Without the digital pinholing, the image resembles the widefield image, but displays higher noise level due to the pixel-dependent gain and noise of the sCMOS camera. c) Optically sectioned image reconstructed from the multi-spot illumination by applying digital pinholing.

a)

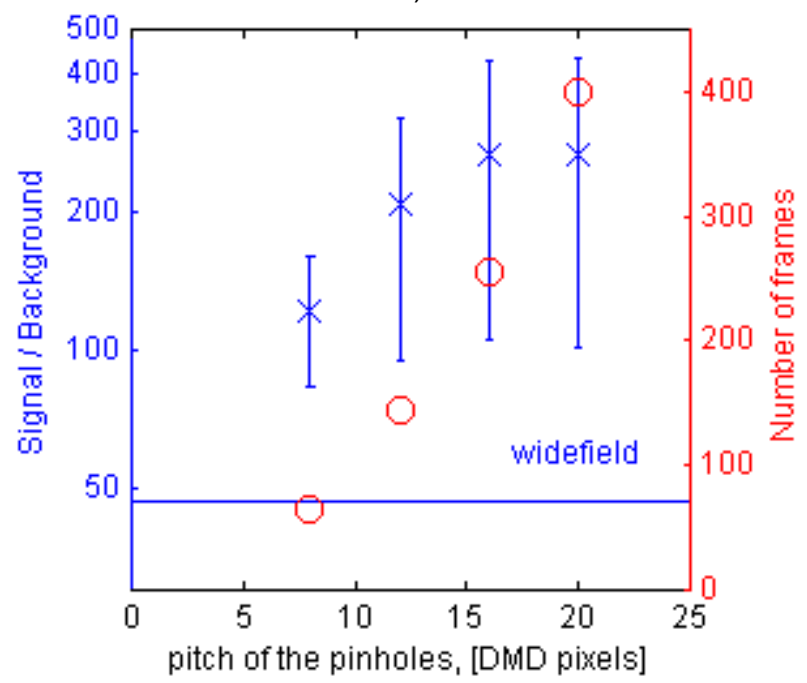

b)

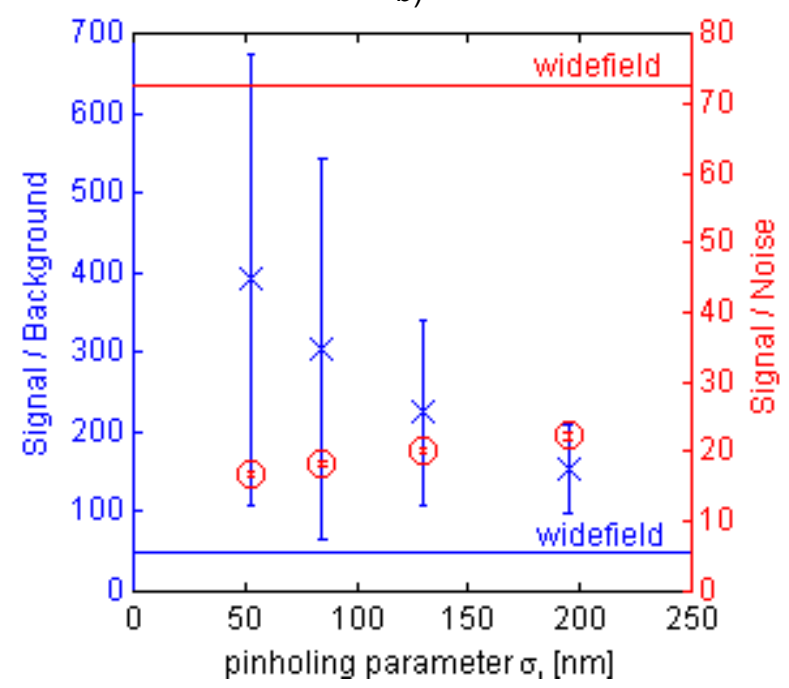

Figure 9. Sectioning capabilities of the DMD-based multi-spot scanning microscope depending on the pitch size (a) and the pinholing parameter $\sigma$ (b). a) The sectioning effect can be improved by increasing the pitch of the projected multi-spot pattern. However, the number of frames required and the image acquisition time depend quadratically on the pitch. A pitch of 12-16 DMD pixels provides a reasonable compromise between sectioning and image acquisition time. The horizontal line indicates the SBR in a widefield image. b) By decreasing the digital pinhole parameter $\sigma$ we enhance the sectioning on the one hand and deteriorate the SNR on the other hand. The optimal value $\sigma \approx 85 \mathrm{~nm}$ corresponds to the pinhole size equal to the first dark ring of the Airy pattern. Horizontal lines indicate the SBR and SNR in a widefield image. 

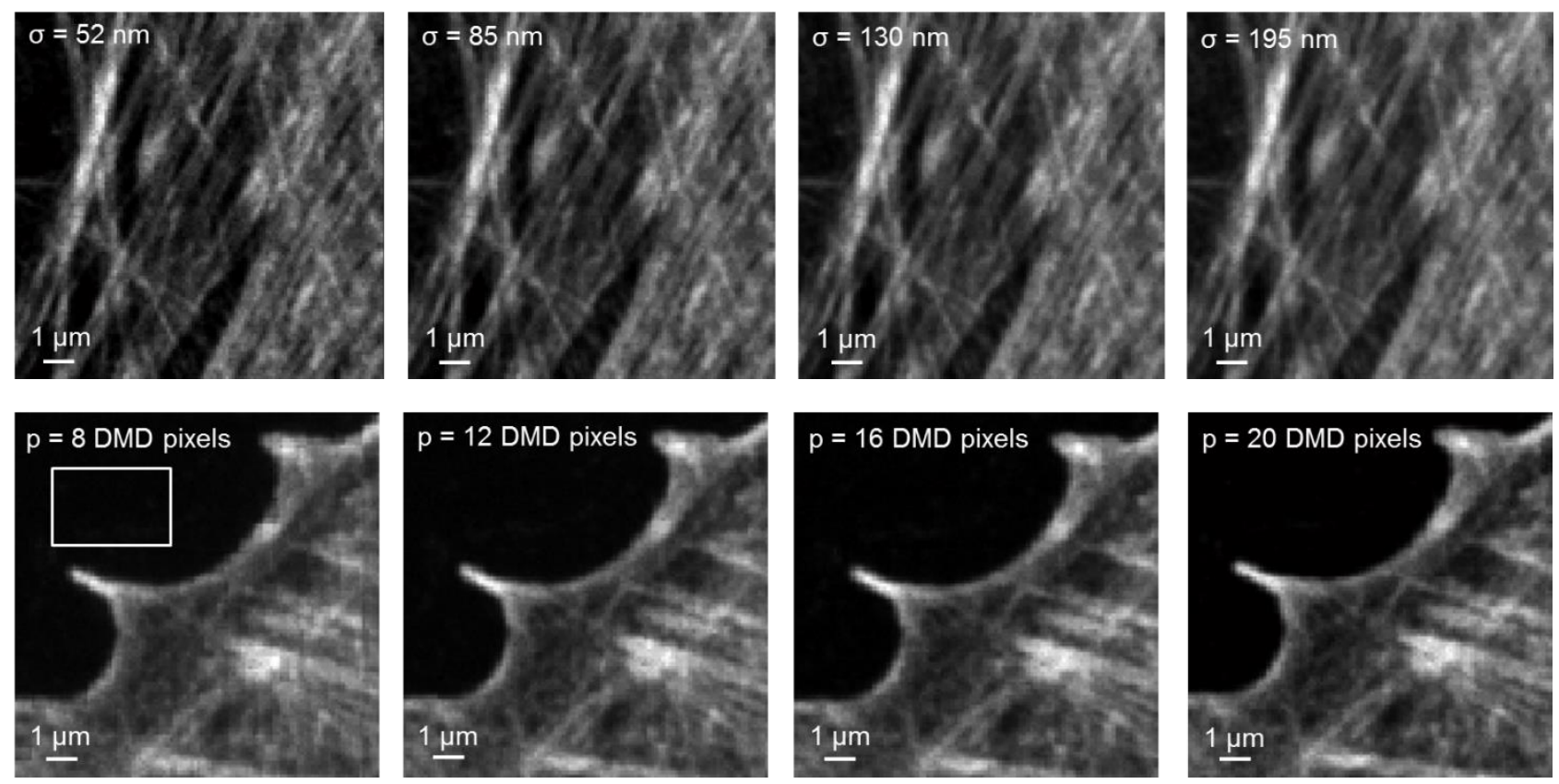

Figure 10. Top row: comparison of the images reconstructed using different values of the pinhole parameter $\sigma$. The images are acquired with the optimal value of the pitch $p=16 \mathrm{DMD}$ pixels, and are displayed with linearly stretched contrast, since there is a large difference in the intensities of the images reconstructed using different $\sigma$ values. The sectioning effect is enhanced with decreasing pinhole parameter $\sigma$. Bottom row: comparison of the images acquired with different pitch values $p$. The images are reconstructed using the optimal pinhole parameter $\sigma=85 \mathrm{~nm}$ and have the same contrast stretching. A small pinhole separation $p=8 \mathrm{DMD}$ pixels results in artefacts in the reconstructed image. A pinhole separation of 12-16 DMD pixels provides optimal results. Further increase of the pitch size leads only to a minor sectioning improvement. The sectioning strength was assessed by the SBR in each image. The signal was estimated as the mean of the $1 \%$ highest intensity pixels and the background as the mean of the background area, which is shown as a white box in the bottom-left image.

\section{OUTLOOK ON THE USE OF PSEUDO-RANDOM PATTERNS}

Pseudo-random sequences were first applied in a spinning disk microscope in 1996 by Juškaitis et al. ${ }^{8}$ to address the issue of inefficient use of the available illumination light in confocal microscopy. Similar types of patterns have been later applied in dual-pass DMD-based microscopes ${ }^{12,23}$. In both spinning disk and DMD-based microscopes the sectioning effect was obtained by illuminating the sample through the aperture mask and detecting the fluorescence light through the same aperture mask (dual-pass setup). The use of pseudo-random patterns in a single-pass configuration with wide-field detection is not straightforward, since it requires more elaborate reconstruction algorithms to retrieve a sectioned image. This is even more complicated when improved lateral resolution, as in SIM, is required. Nonetheless, this option might be advantageous over a dual-pass setup due to the simplified optical implementation. To our knowledge, pseudo-random patterns have not been applied in a single-pass DMD-based microscopes with widefield detection up to date. However, the improvement of lateral resolution by applying speckle illumination was recently demonstrated $^{24,25}$, and optical sectioning with speckle illumination and widefield detection was obtained as well ${ }^{26}$. In case of speckle illumination the information about the illuminating patterns is not available, which leads to computationally demanding reconstruction algorithms and, hence, relatively slow reconstructions. We are aiming to apply pseudo-random illumination patterns in a single-pass DMD-based microscope for both sectioning and lateral resolution improvement. A priori knowledge of the illumination patterns is expected to simplify and speed up the reconstruction. Furthermore, the DMD patterns can be designed in such a way that their sum results in a homogeneous intensity distribution, which is expected to reduce the overall noise level. 


\section{REFERENCES}

[1] Schermelleh, L., Heintzmann, R., and Leonhardt, H., "A guide to super-resolution fluorescence microscopy," J. Cell Biol. 190(2), 165-175 (2010).

[2] Pawley, J.B., [Handbook Of Biological Confocal Microscopy], Springer US, Boston, MA (2006).

[3] Gustafsson, M.J.L., "Surpassing the lateral resolution limit by a factor of two using structured illumination microscopy.," J. Microsc. 198(2), 82-87 (2000).

[4] Heintzmann, R., and Cremer, C., "Laterally modulated excitation microscopy: improvement of resolution by using a diffraction grating," Proc. SPIE 3568, 185-196 (1999).

[5] Cragg, G.E., and So, P.T., "Lateral resolution enhancement with standing evanescent waves.," Opt. Lett. 25(1), 46-48 (2000).

[6] Frohn, J.T., Knapp, H.F., and Stemmer, A., "True optical resolution beyond the Rayleigh limit achieved by standing wave illumination" Proc. Natl. Acad. Sci. U.S.A. 97(13), 7232-7236 (2000).

[7] Gustafsson, M.G.L., Shao, L., Carlton, P.M., Wang, C.J.R., Golubovskaya, I.N., Cande, W.Z., Agard, D. A, and Sedat, J.W., "Three-Dimensional Resolution Doubling in Wide-Field Fluorescence Microscopy by Structured Illumination,” Biophys. J. 94(12), 4957-4970 (2008).

[8] Juškaitis, R., Wilson, T., Neil, M.A.A., and Kozubek, M., "Efficient real-time confocal microscopy with while light sources," Nature 383, 804-806 (1996).

[9] Caarls, W., Rieger, B., De Vries, A.H.B., Arndt-Jovin, D.J., and Jovin, T.M., "Minimizing light exposure with the programmable array microscope.," J. Microsc. 241(1), 101-10 (2011).

[10] Liang, M., Stehr, R.L., and Krause, A.W., "Confocal pattern period in multiple-aperture confocal imaging systems with coherent illumination,” Opt. Lett. 22(11), 751-753 (1997).

[11] Hanley, Q.S., Verveer, P.J., Gemkow, M.J., and Jovin, T.M., "An optical sectioning programmable array microscope implemented with a digital micromirror device," J. Microsc. 196(3), 317-331 (1999).

[12] Heintzmann, R., Hanley, Q.S., Arndt-Jovin, D., and Jovin, T.M., "A dual path programmable array microscope (PAM): simultaneous acquisition of conjugate and non-conjugate images," J. Microsc. 204(2), $119-135$ (2001).

[13] Verveer, P.J., Hanley, Q.S., Verbeek, P.W., Vliet, L.J.V.A.N., and Jovin, T.M., "Theory of confocal fluorescence imaging in the programmable array microscope (PAM)," J. Microsc. 189(3), 192-198 (1998).

[14] Křžžek, P., Raška, I., and Hagen, G.M., "Flexible structured illumination microscope with a programmable illumination array," Opt. Express 20(22), 24585-99 (2012).

[15] York, A.G., Parekh, S.H., Dalle Nogare, D., Fischer, R.S., Temprine, K., Mione, M., Chitnis, A.B., Combs, C. A, and Shroff, H., "Resolution doubling in live, multicellular organisms via multifocal structured illumination microscopy," Nat. Methods 9(7), 749-54 (2012).

[16] Dan, D., Lei, M., Yao, B., Wang, W., Winterhalder, M., Zumbusch, A., Qi, Y., Xia, L., Yan, S., et al., "DMD-based LED-illumination Super-resolution and optical sectioning microscopy," Sci. Rep. 3, 1116 (2013).

[17] Texas Instruments TI DN 2509927, "Using Lasers with DLP DMD technology,” (2008).

[18] De Beule, P.A.A., De Vries, A.H.B., Arndt-Jovin, D.J., Jovin, T.M., "Generation-3 programmable array microscope (PAM) with digital micro-mirror device (DMD)," Proc. SPIE 7932, 79320G (2011).

[19] Chen, X., Yan, B., Song, F., Wang, Y., Xiao, F., and Alameh, K., "Diffraction of digital micromirror device gratings and its effect on properties of tunable fiber lasers," Appl. Opt. 51(30), 7214-20 (2012).

[20] Zwier, J.M., Van Rooij, G.J., Hofstraat, J.W., and Brakenhoff, G.J., "Image calibration in fluorescence microscopy," J. Microsc. 216(1), 15-24 (2004).

[21] Boreman, G.D., [Modulation transfer function in optical and electro-optical systems] , SPIE Press, 79-84 (2001).

[22] Goodman, J.W., [Introduction to Fourier Optics], The McGraw-Hill Companies, 138-144 (1996).

[23] Jiang, S., and Walker, J., "Differential high-speed digital micromirror device based fluorescence speckle confocal microscopy," Appl. Opt. 49(3), 497-504 (2010).

[24] Mudry, E., Belkebir, K., Girard, J., Savatier, J., Le Moal, E., Nicoletti, C., Allain, M., and Sentenac, A., "Structured illumination microscopy using unknown speckle patterns," Nature Photon. 6(5), 312-315 (2012).

[25] Min, J., Jang, J., Keum, D., Ryu, S.-W., Choi, C., Jeong, K.-H., and Ye, J.C., "Fluorescent microscopy beyond diffraction limits using speckle illumination and joint support recovery," Sci. Rep. 3, 2075 (2013).

[26] Ventalon, C., and Mertz, J., "Quasi-confocal fluorescence sectioning with dynamic speckle illumination," Opt. Lett. 30(24), 3350-3352 (2005). 